\title{
Der Werth des Augustalis Kaiser Friedrichs II.
} Von

\author{
Adolf Schaube.
}

I. Kritik der Ausführungen Winkelmanns. 1. Allgemeines, 2. Ein Schreibfehler und seine Folgen. 3. Der Ansatz des Silberwerthes. 4. Verrechnung der Prägekosten und des Schlagschatzes. 5. Höhe der Gebühr bei Prägungen für private Rechnung. 6. Das gesetzliche Werthverhältnis der uncia tarenorum und der uncia augustalium. 7. Ansatz des sizilischen Pfundes und des Tarì. 8. Berechnung des Durchschnittsgewichts des Augustalis. II. Positiver Theil. 9. Ermittelung des wahren Gewichts der sizilischen Unze und ihrer Theile. 10. Ermittelung des Metallwerthes der Goldunze und ihrer Theile. 11. Ermittelung des Kosten. und Nennwerthes. 12. Tabelle und Bemerkungen zu den Ergebnissen.

1. In der anziehenden Abhandlung über die Goldprägungen Kaiser Friedrichs II., die E. Winkelmann in diesen Blättern veröffentlicht hat 1), hat er als Historiker ein besonderes Gewicht darauf gelegt, den Werth der friedericianischen Goldmünzen zu bestimmen; mit Recht hebt er hervor, dass es gelte, durch solche Vermittelung einen festen Massstab für die Beurtheilung der Zahlenangaben auf den verschiedensten Gebieten der öffentlichen Verwaltung wie des wirthschaftlichen Lebens zu gewinnen ${ }^{2}$ ). So gründlich und mühsam nun auch die Untersuchung geführt ist ${ }^{3}$ ), so interessant manche ihrer Ergebnisse sind, so muss doch behauptet werden, dass gerade die Ergebnisse, die sich auf den ron dem Verf. selbst als besonders wichtig bezeichneten

1) $\mathrm{XV}, 401-440$.

2) S. 431 .

s) Eine sehr sorgtältige Studie wird sie in der hist. Zeitschr. 74, S. 169 genannt.

Mittheilungen XVI. 
Punkt beziehen, der Sicherheit, die der Verf. für sie zu erreichen wünschte, damit sie ihm und anderen für künftige Arbeiten als feste Grundlage dienen köunten, nur allzusehr entbehren. Schon ein prüfender Blick auf die von Winkelmann am Schlusse seiner Abhandlung gegebene tabellarische Zusammenstellung der hauptsächlichsten Rechnungsergebnisse genügt, um diese Thatsache ausser $\mathrm{Z}_{w}$ eifel zu stellen. Der Gehalt an Feingold ist hier für die uncia augustalium (mit $18,28 \mathrm{Gr}$.) geringer angegeben als für die uncia tarenorum (nit 18,37 Gr.); noch mehr bleibt ihr Gewicht und damit ihr Gehalt an Silber hinter dem der uncia tarenorum zurück $(21,69 \mathrm{Gr}$. Gewicht gegen $27,12 \mathrm{Gr}$.); dabei soll aber ihr Metallwerth um 1,28 M. höher gewesen sein als der der uncia tarenorum $(52,88$ gegen 51,60 M.). Das ist ein unleugbarer Widerspruch, den der Verf. übersehen hat, offenbar in der Freude darüber, dass die Probe, der er seine Ergebnisse unterworfen hat, zu stimmen schien ${ }^{1}$ ). Wenn ich diese Freude auch zerstören muss, so bin ich doch überzeugt, dass der Verf. der erste sein wird, die Aufdeckung der Irrtümer, die ihm untergelaufen sind, willkommen zu heissen; oft genug schon hat er bewiesen, dass es ihm allein um Feststellung der Wahrheit und nicht um Festhaltung der eigenen Meinung $\mathrm{zu}$ thun ist.

2. Ich beginne mit einem Fehler trivialster Art, der sich in Winkelmanns Berechuung des Metallwerthes des Augustalis eingeschlichen hat. Nach seinem Ansatz enthielt der Augustalis neben 4,57 Gr. Feingold 0,585 Gr. Feinsilber. Inden Winkelmann nun das Pfund Feinsilber zu 48 Mark rechuete, also rund auf $100 \mathrm{Gr}$. 10 Mark, auf 1 Gr. 0,10 Mark, wollte er die 0,585 Gr. Feinsilber offenbar mit $0,05 \mathrm{M}$. in Ansatz bringen; in Wirklichkeit hat er sie aber infolge eines lapsus calami mit 0,50 M. in Ansatz gebracht ${ }^{2}$ ). Damit ist der Werth des Feinsilbers zwanzigmal so hoch genommen, als Winkelmann ihn hat ansetzen wollen, der Metallwerth des Augustalis um 45 Pfennige, der der uncia augustalium um 1,80 M. höher geworden, als es nach den sonstigen Aufstellungen Winkelinanns der Fall gewesen wäre.

Der Schreibfehler hat nun auch weitere Folgen nach sich gezogen. Um das Ergebnis auf seine Richtigkeit zu prüfen, zog Winkelmann die Angabe der Münzerordnung heran, nach der das Metall der uncia augustalium der Regierung auf 27 Tarì 18 Gran zu stehen kam. Natürlich stimmte die Probe nicht. Nicht ahnend, dass der Grund in der Tücke des Objekts zu suchen sei, glaubte er die Fehlerquelle

I) Mittheil. XV, 437.

2) S. 435. 
in den Voraussetzungen der Rechnung erblicken zu müssen und konjizierte XXVIII statt XXVII Tari ${ }^{1}$ ), wodurch die unerwünschte Differenz allerdings ziemlich vollständig beseitigt wurde. Selbstverständlich entfällt nunmehr nach Aufdeckung der wahren Fehlerquelle jeder Grund zu der vorgenommenen Aenderung an dem überlieferten Texte.

Es entfällt ferner jetzt schon eine allgemeinere Folgerung, die Winkelmann ziehen zu können geglaubt hat. In Folge jenes Irrthums nimmt er den Unterschied zwischen Metallwerth und Verkehrswerth der uncia augustalium auf nur $2,48 \mathrm{M}$. an, während er denselben Unterschied bei der uncia tarenorum auf $3,77 \mathrm{M}$. berechnet hat. Danach hätte sich also die kaiserliche Regierung bei der Ausprägung der Augustalen mit einem viel kleineren Gewinne als bei Prägung der Goldtarì begnügt und die sonst bei ihr deutlich genug hervortretende Fiscalität der ,höchst wünschenswerthen Gleichheit im Verkehrswerthe der beiden Unzenarten" zum Opfer gebracht 2). Auch das hat nur der Schreibfehler verschuldet; ohne ihn würde Winkelmann den Unterschied zwischen Metall- und Verkehrswertb der uncia augnstalium auf 4,28 M. berechnet und damit also keinen Grund zu der Annahme einer bei der Prägung der Augustalen vorliegenden Abweichung von den sonstigen Regierungsmaximen des Kaisers gefunden haben.

3. Ich würde den Schreibfehler schwerlich so leicht entdeckt haben, wenn ich nicht zuerst auf einen zweiten Irrtum des Verfassers, einen Irrtum methodischer Art, aufmerksam geworden wäre. Winkelmann setzt das Pfund Feinsilber mit dem Werthe von $48 \mathrm{M}$. an, d. h. mit dem Marktpreis, den das Silber am Tage der Niederschrift des bezüglichen Abschnitts seiner Abhandlung (nach eigener Angabe am 24. November 1893) besass ${ }^{3}$ ). Dass dieser Preis ein sehr unsicherer sei, bemèrkt Winkelmann selbst; wie konnte er dann aber hoffeu, auf so unsicherer Grundlage zu sicheren Ergebnissen zu kommen? Wenn er nun zwanzig Jahre früher an seine Untersuchung herangegangen wäre, als der Preis des Silbers noch ein weit höherer war? Es ist klar, dass es unmöglich ist, bei einer wissenschaftlichen Untersuchung dieser Art sich auf den schwankenden Augenblickspreis des Weltmarktes zu stützen. Man bedenke, dass es sich u. a. darum handelt, zu ermitteln, zu welchem Preise die kaiserliche Münze unter Friedrich II. das Silber, dessen sie zur Ausprägung ihrer Goldmünzen bedurfte, erstanden, welchen Gewinn die Regierung aus diesen Prägungen gezogen hat,

1) S. 437 .

2) S. 436,438 .

8) S. 433 . 
wie das Werthverhältnis verschiedener Legierungen zu beurtheilen ist. Dafür kann doch nichts anderes, als der Silberpreis der damaligen Zeit als massgebend angesehen werden, der Silberpreis, wie er aus dem damals herrschenden Werthrerhältnis von Silber und Gold abzuleiten ist. Wenn ich Goldmünzen verschiedener Zeiten mit einander vergleiche, so kann ich das nur in der Weise thun, dass ich die ausser dem Feingold in ihnen enthaltenen Elemente nach dem Werthverhältnis, in dem sie zu ihrer Zeit zum Golde standen, abschätze.

Winkelmann rechnet also mit einer Werthrelation zwischen Gold und Silber von 29:1, während das unseren Währungsverhältnissen noch zu Grunde liegende Werthverhältnis bekanntlich $15 \frac{1 / 2}{2}: 1$ ist. Im Mittelalter aber war dies Verhältnis für das Silber noch erheblich günstiger. Schon Soetbeer hat im Jahre 1880 das Endergebnis einer längeren Uutersuchung über diesen Gegenstand dahin gezogen, dass im ganzen und durchschnittlich genommen, sich die Werthrelation vom 13. bis 15. Jahrhundert innerhalb der Grenzen von 10 bis 11:1 gehalten habe ${ }^{1}$ ). Einige Jahre später hat er seine Ansicht insofern etwas abgeändert, als er nunmehr für die Zeit rom 9. bis zum Anfang des 16. Jahrhunderts in Europa annahm, dass es sich i!n grossen und ganzen zwischen den Grenzen von 12:1 und 10:1 bewegt habe ${ }^{2}$ ). Schon vor ihm hatte Blancard ein Werthverhältnis von 12,5:1 angenommen und seinen Berechnungen zu Grunde gelegt ${ }^{3}$ ); indessen hat ein anderer französischer Forscher, Marchéville, auf Grund umfassender und genauer Prüfung des in Betracht kommenden Materials neuerdings für die Mitte des 13. Jahrhunderts eine Werthrelation von 10:1 berechnet ${ }^{4}$ ). Auf die Details kann natürlich an dieser Stelle nicht eingegangen werden; ich begnüge mich mit dem Hinweise darauf, dass im Jahre 1252, als in Florenz die Prägung von Goldmünzen begann, das Werthrerhältnis in dieser Stadt auf 10,7:1 stand ${ }^{5}$ ) und dass Berechnungen, die ich für den Anfang des 13. Jahrhunderts angestellt habe, in ihrem Ergebnis eher unter als über das Niveau von 10:1 geführt haben 6). Danach halte ich es für gerechtfertigt, für die Zeit

1) Soetbeer A.: Edelmetallproduktion in Petermann's Mittheilungen. Ergänz.-Bd. 13, Gotha 1880 Nr. 57, S. 120.

2) Materialien zur Erläuterung der wirthschattlichen Edelmetallverhältnisse. 2. Ausg. Berlin 1886 S. 21.

3) Blancard, L. in seinen Aufsätzen in der Revue numism. und dem aus diesen erwachsenen Essai sur les monnaies de Charles I, Comte de Provence. Paris 1879.

4) Annuaire de la société de numism. Année 1890 p. 158.

s) Pagnini : Della Decima. T. I, tavola 4. Lisb. e Lucca $1765 / 6$; dazu Soetbeer, Materialien S. 22.

$\left.{ }^{6}\right)$ Vgl. hiezu nunmehr auch die Abhandlung von K. Th. v. Inama-Sternegg: 
der Ausprägung von Augustalen, die 1231 begann, für das Werthverbältnis von Gold und Silber den runden. Betrag von 10:1 zu Grunde zu legen und danach das Pfund Feinsilber mit 139,2 M. anzusetzen. Sollten wir wider Erwarten damit irren, so dass etwa einer Relation von 11:1 der Vorzug zu geben wäre, so würde sich der Fehler, den wir gemacht haben, immerhin nur auf $2 \frac{1}{2}$ Pfennig für das Gramm Feinsilber belaufen, während Winkelmanns Ansatz des Silberwerthes gegenüber dem unsrigen einen entsprechenden Unterschied von 18 Pfennigen bedingt. Mit seinem Ansatz des Silberwerthes hat Winkelmann den Metallwerth der uncia augustalium um 40 Pfennige, den der uncia tarenorum um $1,30 \mathrm{M}$. zu niedrig berechnet.

4. Ein weiterer Irrthum Winkelmanns liegt in der Verrechnung der Prägekosten vor. Nach der Angabe der Münzerordnung kam der Regierung die Unze, wenn sie sich aus Augustalen zusammensetzte, auf 27 Tarì 18 Gran, und falls sie durch Goldtarì gebildet wurde, auf 28 Tarì $2 / 3$ Gran zu stehen, während sie in beiden Fällen natürlich zum vollen Nennwerthe der Unze, also zu 30 Tarì, ausgegeben wurde. Falls Private für ihre Rechnung Augustalen oder Goldtarì von der kaiserlichen Münze prägen liessen, so hatten sie an Prägekosten auf die Unze $4 \frac{1}{2}$ Gran zu vergüten und ausserdem eine Abgabe von $15 \frac{1}{2}$ Gran an den Fiskus, im ganzen also 1 Tarì, zu entrichten. Winkelmann erblickt nun in jener ersten Angabe der Münzerordnung den Metallwerth der Unze und bezeichnet die Differenz zwischen diesem Werthe und den 30 Tarì, dem Umlaufswerthe der Unze, als den Vortheil, den die Regierung aus der Prägung gezogen habe. Daraus berechnet er nunmehr den Verkehrswerth der Unze, indem er ausser dem Metallwerthe und dem der erwähnten Differenz gleichgesetzten Gewinn der Regierung ausserdem noch die Unkosten der Prägung in Rechnung setzt ${ }^{1}$ ). Winkelmann hat dabei den Widerspruch übersehen, der darin liegt, dass ja dann die Unze zu einem höheren Betrage als mit 30 Tarì ausgegeben sein müsste; er hat übersehen, dass nach seiner Auffassung die Prägekosten nothwendig in der Differenz zwischen dem von der Münzerordnung angegebenen Metallwerthe und dem Nominalwerthe der Unze mit enthalten sein müssen. Es ist also von seinem Standpunkt aus auch nicht zutreffend, den ganzen Betrag dieser Differenz als Gewinn der Regierung, als Schlagschatz zu bezeichnen. Hält man aber letzteres für richtig, wie ich es thue und unten begründen werde, so darf man doch die Prägekosten eben-

Die Goldwährung im deutschen Reiche während des Mittelalters in der Zeitschr. für Social- und Wirthschaftsgesch. III, 19.

1) S. 434 . 
sowenig ausserhalb dieser beiden Faktoren suchen, dann sind sie vielmehr in der Angabe des , valet' der Münzerordnung, in der Angabe, wie hoch der Regierung die Unze geprägter Goldmünzen zu stehen kam, mit enthalten; wir haben es dann nicht mit der Angabe des reinen Metallwerthes, sondern der des Kostenwerthes der Unze zu thun. Jedenfalls musste also bei Winkelmann die besondere Verrechnung der Prägekosten unterbleiben; um den Betrag derselben (39 Pfennige) hat er demnach den Verkehrswerth der Unze zu hoch angesetzt.

Noch eine weitere Ungenauigkeit ist bezüglich des Ansatzes des Gewinnes der Regierung bei der Berechnung des Verkehrswerthes der uncia tarenorum untergelaufen. Für die 2 Tarì weniger $2 / 3$ Gran der Münzerordnung, die er als Gewinn der Regierung ansieht, bringt er den vorher von ihm in Höhe von 1,72 M. ermittelten Metallwerth des Goldtarì in Ansatz, während doch klar ist, dass sich die Angaben der Münzerordnung nur auf den Verkehrswerth, den Nennwerth, beziehen können. Wenn dieser Werth auch noch nicht ermittelt war, so liess sich dieser Mangel doch durch eine einfache Rechnungsoperation ausgleichen 1). Der Fehler fällt um so mehr auf, als sich Winkelmann in dieser Beziehung nicht konsequent geblieben ist, da er bei der uncia augustalium ganz richtig mit dem Nominalwerth des Goldtarì, den er zu 1,84 M. ansetzt, gerechnet hat. Die 2 Tarì 2 Gran hier sind mit 3,88 M., die 1 Tarì $19 \frac{1}{1} 3$ Gran dort mit nur 3,38 M. berechnet ${ }^{2}$ ); die Differenz beträgt also 50 Pfennige, während sie nur 25 hätte betragen dürfen. Hätte Winkelmann in beiden Fällen dasselbe Prinzip der Berechnung angewandt, so wäre er zu einer genauen Uebereinstimmung des Verkehrswerthes beider Unzen gar nicht gelangt.

5. Bezüglich der Höhe der Abgabe, die bei Goldprägungen für private Rechnung zu entrichten war, hat Winkelmann eine zweite Korrektur der Münzerordnung für nothwendig erachtet, da ihm diese Abgabe im Vergleich zu dem Gewinne, den die Regierung aus der Goldprägung für eigene Rechnung zog, zu niedrig erschien. Er vermuthet, dass statt grana $\left.15^{1}\right|_{2}$ zu lesen sei tarenum unum grana $15^{1 / 2}{ }^{3}$ ). Indessen kann man nicht sagen, dass Winkelmann mit dieser Konjektur glücklicher gewesen wäre als mit der ersten. Zunächst hat er nicht bemerkt, dass diese beiden Konjekturen sich unter einander durchaus nicht vertragen. Wenn der Metallwerth der uncia augustalium nach der ersten Konjektur 28 Tarì 18 Gran, der Gewinn der Regierung

1) Vgl. unten S. 561.

2) S. 437 und 434 .

9) Ebenda und 421 A. 1. 
demnach (ich sebe hier, da ich mich auf den Boden Winkelmanns stelle, von der Differenz wegen der Prägekosten ganz ab) nur 1 Tarì 2 Gran betragen haben soll, wie sollte da die Regierung bei privaten Prägungen einen Betrag von fast doppelter Höhe (1 Tarì $\left.15^{1}\right|_{2}$ Gran Entschädigung für entgangenen Gewinn und $41 / 2$ Gran Prägekosten, also 2 Tarì im ganzen) haben erheben können? Wie hätte sich ein Kaufmann dazu verstehen sollen, der Münze Metall im Werthe von 28 Tarì 18 Gran zu liefern und dann noch 2 Tarì zuzuzahlen, um dafür 4 Augustalen im Nominalwerthe von 30 Tarì zurückzuerhalten? Der Kaufmann, der in der Münze für seine Rechnung prägen liess, sollte doch keinen Verlust erleiden. Dieser Umstand spricht gegen die zweite Konjektur Winkelmanns aber auch dann, wenn wir von ihrer Unvereinbarkeit mit der ersten absehen. Denn der Metallwerth der uncia tarenorum belief sich auf 28 Tarì $\left.2\right|_{3}$ Gran; hatte der Private, der für eigene Rechnung prägen liess, 2 Tarì für die aus Goldtarì bestehende Unze an den Fiskus zu entrichten, so erlitt er dem Nominalwerth der Unze gegenüber einen wenn auch nicht allzuerheblichen Verlust, den auf sich zu nehmen er indessen keinerlei Veranlassung haben kounte. Uebrigens wird die Unhaltbarkeit der zweiten Konjektur Winkelmanns meines Erachtens schon durch den vollen Wortlaut der betreffenden Stelle der Münzerordnung selbst dargethan: Consuevit curia recipere pro qualibet uncia tam tarenornm quam augustalium, que laboratur in predictis siclis, grana $\left.15^{1}\right|_{2}$. Verumtanen mercator, qui facit laborari aurum suum in siclis ipsis, preter grana quindecim et medium debet solvere alia grana $\left.4^{1}\right|_{2}$ pro qualibet uncia ... pro expensis, que fiunt in labore uncie cuiuslibet etc. Es müsste also das tarenum unum, das Winkelmann ergänzen will, an zwei verschiedenen Stellen dieses Abschnitts der Münzerordnung ausgefallen sein. Ja noch mehr. Es wird weiter bestimmt, dass der Kaufmann die Wahl haben solle, ob er die $\left.4\right|_{2} ^{1}$ Gran Prägekosten zahlen oder die nothwendigen Ausgaben lieber aus eigenen Mitteln bestreiten wollte; und ausdrücklich wird wiederum hinzugefügt: Et tunc non debet curie 4 predicta grana et $\left.1\right|_{2}$, set quindecim grana et medium tantum pro qualibet uncia, et mercator ipse facit expensas de suo proprio ${ }^{1}$ ). Damit entfällt für die Richtigkeit der Vermuthung Winkelmanns auch jede äussere Möglichkeit. Bei der Beurtheilung der Höhe des Gewinnes, den, wie die Regierung, auch der Kaufmann machte, der für eigene Rechnung prägen liess, müssen wir uns hüten, einen modernen Massstab anzulegen. Mit unseren Begriffen verträgt sich die

1) Acta Imperii ed. Winkelmann $I, 766$. 
Berechnung eines so erheblichen Schlagschatzes bei der Ausprägung von Goldmünzen, wie er sich aus den Angaben der Münzerordnung unzweideutig ergiebt, überhaupt nicht. Und nun erwäge man, dass der Zinsfuss, dass überhaupt jede Art des Gewinns, mit dem der Kaufmann rechnete, im 13. Jahrhuudert ganz erheblich höher war als in unseren Tagen und man wird an einem Gewinn von etwa $3{ }_{3}^{2}$ Prozent, den der Kaufmann machte, der auf eigene Rechnung Augustalen prägen liess, nichts Auffälliges mehr finden.

6. Für die Untersuchung ist die Vergleichung des Werthes des Augustalis mit dem des Goldtarì von erheblicher Wichtigkeit. Ein Augustalis wog 6 Tarì und hatte einen Nominalwerth von $\left.{ }_{4}\right|_{4}$ Goldunze $=\left.7{ }^{1}\right|_{2}$ Goldtarì; das geht aus den Angaben, die wir aus dem 13. Jahrhundert hierüber besitzen, mit Sicherheit hervor. Die Frage ist, ob auch der innere Werth des Augustalis ein entsprechender war und ob er nicht mehr oder minder von dem inneren Werthe, den $\left.7^{11}\right|_{2}$ Goldtarì besassen, abwich. Winkelmann hat, um diese Frage beantworten zu können, einen langwierigen Weg einschlagen zu müssen geglaubt, einen Weg, der dabei doch zu einem gesichertem Ergebnis nicht geführt nat. Er kommt zu dem Resultat, dass für die uncia augustalium und die uncia tarenorum völlige Gleichheit des Goldgehalts beabsichtigt gewesen sei; jene hätte 18,28, diese 18,37 Gramm an Feingold enthalten.

Schon am Eingang haben wir hervorgehoben, dass dieses Resultat dem von Winkelmann für den Metallwerth gewonnenem Ergebnisse widerspricht. Winkelmann hat nicht erkannt 1), dass uns ja die Angaben der Münzerordnung selbst in den Stand setzen, das gegenseitige, gesetzmässige Verhältnis der uncia augustalium und der uncia tarenorum in Bezug auf Goldgehalt wie Metallwerth mit voller Klarheit und Genauigkeit festzustellen. Demgemäss bin ich auch gar nicht der Meinung, die Winkelmann bei Beginn seiner Abhandlung äussert 2), dass die Ueberlieferung zur Lösung unserer Aufgabe nicht ausreiche; ich behaupte vielmehr, dass wir bei wenigen Münzen des Mittelalters so genaue zeitgenössische Angaben haben, wie gerade beim Augustalis und dass wir in diesem Falle einer Untersuchung der Münzen selbst gar nicht bedürfen, um zu einem zuverlässigen Ergebnis zu gelangen. Immerhin ist auch letztere Untersuchung der Kontrolle wegen, die sie ermöglicht, nicht ohne ihren besonderen Werth.

Die uncia augustalium, in 4 Augustalen ansgeprägt, wog 24 Tarì, während die uncia tarenorum, die zu 30 Goldtarì ausgeprägt wurde,

i) Mittheil. XV, 428.

?) S, 402 . 
auch 30 Tarì wog. Dem Mindergewicht der uncia augustalium stand aber ein zunächst relativ erheblich stärkerer Gehalt an Feingold gegenüber; zur Prägung der Augustalen wurde $\left.20^{1}\right|_{2}$ karätiges, zur Prägung der Goldtarì nur $\left.16^{1}\right|_{3}$ karätiges Gold rerwendet, während die Legierung in beiden Fällen mit zwölflöthigem Silber erfolgte.

Daraus folgt, dass die uncia augustalium unter ihren 24 Gewichtstarì 201/2 Tarì Feingold enthielt; die uncia tarenorum dagegen musste mit ihren $24+6$ Gewichtstari $\left.16^{1}\right|_{3}$ Tarì Feingold + ein Viertel dieses Betrages, also $\left.4\right|_{12}$, in Summa $\left.20^{5}\right|_{12}$ Tarì Feingold enthalten. Damit stehen einander $\left.20^{6}\right|_{12}$ in der uncia augustalium, $\left.20^{5}\right|_{12}$ in der uncia tarenorum enthaltene Tarì Feingold gegenüber und wir haben damit schon das wichtige und interessante Ergebnis gewonnen, dass nicht völlige Gleichheit des Goldgehalts der beiden Unzen beabsichtigt war, dass noch weniger der Goldgehalt der uncia augustalium hinter dem der uncia tarenorum um ein Geringes zuräckblieb, sondern dass die in Augustalen ausgeprägte Unze die in Goldtarì ausgeprägte um ein Quantum Gold im Gewichte von ${ }_{1 / 12}$ Tarì übertraf, dass also der einzelne Augustalis $\left.{ }^{1}\right|_{48}$ Tarì $=\left.1^{2}\right|_{3}$ Gran Feingold $\mathrm{mehr}$ enthielt, als die ihm in Verkehrswerth entsprechenden $\left.71\right|_{2}$ Goldtarì.

Der etwas grössere Goldgehalt der uncia augustalium hat nun freilich gar nichts Auffälliges, da bei ihrem geringeren Gewicht ihr Silbergehalt ein sehr viel niedrigerer war als der der uncia tarenorum. Auf die 24 Gewichtstarì der uncia augustalium entfallen $\left.3^{1}\right|_{2}$ Gewichtstarì zwölflöthigen Silbers, oder wenn wir dasselbe gleich in seine Bestandtheile zerlegen, $\left.{ }^{7}\right|_{8}$ Tarì Kupfer und $\left.{ }^{21}\right|_{8}$ Tarì Feinsilber. Auf die $24+6$ Gewichtstarì der uncia tarenorum entfallen $\left.72\right|_{3}$ Tarì zwölflöthigen Silbers + ein Viertel dieses Betrages: $\left.92\right|_{12}+\left.{ }^{23}\right|_{12}=\left.115\right|_{12}$ Tarì zwölflöthigen Silbers, das sind $\left.{ }^{115}\right|_{48}$ Tarì Kupfer und $\left.{ }^{345}\right|_{48}$ Tarì Feinsilber. Wenn wir uns nur mit dem Feinsilber beschäftigen, so stehen also einem Silbergehalt von $\left.{ }^{126}\right|_{48}$ Tarì der uncia augustalium $\left.{ }^{345}\right|_{48}$ Tarì der uncia tarenorum gegenüber; letztere übertraf die erstere $2,74 \mathrm{mal}$ an Silbergehalt; die uncia augustalium enthielt $\left.4^{27}\right|_{48}$ Gewichtstarì an Feinsilber weniger als die uncia tarenorum, sodass der einzelne Augustalis ungefähr um 1 Tarì 3 Gran an Silbergehalt hinter den seinem Verkehrswerthe entsprechenden $71_{2}$ Goldtarì zurückblieb.

Stellen wir diese Ergebnisse der Uebersichtlichkeit wegen in einer kleinen Tabelle zusammen, so enthielten in Gewichtstarì ausgedrückt die uncia a ugustalium die uncia tarenorum
an Feingold: $\left.20^{6}\right|_{12}$
an Feinsilber: $\left.2^{10}\right|_{16}$ $\left.20^{5}\right|_{12}$
an Kupfer:

$\frac{\left.42\right|_{48}}{24}$ $\left.2^{19}\right|_{48}$ 
So steht bei der uncia augustalium, verglichen mit der uncia tarenorum, einem Plus von $1_{12}$ Gewichtstarì in Gold ein Minus von $\left.4^{9}\right|_{16}$ Tarì im Silber und $\left.1^{25}\right|_{48}$ Tarì in Kupfer gegenüber, was ihrem geringeren Gewicht von 6 Tarì entspricht. Diesem geringeren Gewicht entspricht natïrlich auch ein geringerer Metallwerth; denn das erhebliche Minus an zwölflöthigem Silber wird selbstverständlich durch das kleine Plus an Gold beiweitem nicht ausgeglichen. Legen wir dem Minus an Silber mit Rücksicht anf die $\left.1^{25}\right|_{48}$ Tarì in Kupfer den geringen Betrag von $\left.{ }^{1}\right|_{18} \mathrm{zu}$, so erhalten wir $\left.4^{28}\right|_{48}=4^{7 / 12}$ Tarì in Silber. Reduzieren wir diese auf Gold, indem wir die Werthrelation von 10:1 zu Grunde legen, so würde sich ein Betrag von $\left.{ }^{55}\right|_{120}=\left.11\right|_{24}$ Tarì in Gold ergeben. Wenn wir davon noch das Plus von $\left.{ }^{1}\right|_{12}$ Tarì in Abzug bringen, so gelangen wir zu dem Ergebnisse, dass der Metallwerth der uncia augustalium, in Feingold berechnet, hinter dem der uncia tarenorum um $\left.{ }^{9}\right|_{24}=\left.3\right|_{8}$ Tarì, das sind $\left.71\right|_{2}$ Gran zurückblieb, dass also der einzelne Augustalis den ihm im Verkehrswerthe gleichgestellten $\left.7\right|_{2}$ Goldtarì gegenüber um 1,875 Gran Feingold minderwerthig war. 7. Auch die Art und Weise, wie Wiukelmann die Zurückführung der im sizilischen Königreich gebräuchlichen Gewichte auf unser Gewichtssysten vorgenommen hat, kann ich nicht für zuverlässig genug erachten ${ }^{\mathbf{1}}$ ). Er ist von dem Durchschnittsgewicht des Augustalis ausgegangen, das er aus dem thatsächlichen Gewicht einer erheblichen Zahl von Augustalen ermittelt hat; offenbar hat er keine Mühe gescheut, um in den Besitz einer möglichst grossen Zahl thatsächlicher Gewichtsangaben zu gelangen und die Untersuchung in dieser Beziehung auf eine möglichst gesicherte Grundlage zu stellen. Er hat dann, Blancard folgend, dem so ermittelten Durchschnittsgewicht ein Prozent als Ersatz dessen, was den Münzen durch Abnutzung verloren gegangen sei, zugerechnet. Auf diesem Wege gelangt er dazu, die Unze zu 26,73, das Pfund zu $320,76 \mathrm{Gr}$. zu bestimmen. Winkelmann meint nun selbst, dass dieses Ergebnis noch nicht als unbedingt richtig gelten könne, hält es vielmehr wegen der starken Annäherung dieser Werthe an des altrömische Pfund für in hohem Grade wahrscheinlich, dass das Goldpfund des sizilischen Königreiches mit dem altrömischen Pfunde von $325,44 \mathrm{Gr}$. identisch gewesen sei, woraus sich für die Unze dann ein Gewicht von 27,12 Gr. ergeben würde. Dass das nur eine Hypothese ist, verkennt Winkelmann auch selbst nicht, doch meint er eine Stütze für dieselbe darin gefunden $\mathrm{zu}$ haben, dass das Gewicht des Goldtari thatsächlich $d \mathrm{em}$ des dreissigsten Theiles der so ermittelten Unze $(0,90 \mathrm{Gr}$.)

1) S. $414-416$. 
entsprochen habe. Mir freilich will es scheinen, dass diese Stütze noch erheblich unsicherer ist als das, was durch sie gestützt werden soll. Die Zahl der Goldtarì nämlich, für die Winkelnıann Gewichtsangaben besass, war nur eine ziemlich beschränkte und nimmermehr wäre es möglich gewesen, aus diesen Stücken allein, die theils einfache, theils mehrfache Tarì darstellen, ein bestimmtes Durchschnittsgewicht abzuleiten. Ihr Gewicht bewegt sich innerhalb der Grenzen von 3,74 und 0,93 Gr. und 6 von den 11 Stücken, die Winkelmann anführt, halten sich innerhalb der engeren Grenzen von 1,25 bis 1,43 Gr. Dass damit nichts zu machen ist, liegt auf der Hand. Dass es sich erklärt aus der Thatsache, dass diese Goldtarì nicht zugezählt, sondern zugewogen zu werden pflegten, damit bin ich mit Winkelmann vollständig einverstanden. Einverstanden bin ich auch damit, dass nur das im Gewichte leichteste unter diesen Stücken (mit 0,93 Gr.) als ein einfacher Goldtarì anzusehen ist 1). Aber auch das Gewicht dieses einen Stückes stimmt mit dem postulierten Gewichte nicht völlig überein; Winkelmann sieht es als einen zufällig gut gemessenen Goldtarì an und beruft sich im übrigen auf einige salervitaner Stücke der normannischen Zeit im Gewichte von 0,83 bis 0,90 Gr. und eine Goldmünze Karls I. im Gewichte von 0,86 Gr. ${ }^{2}$ ). Das sind doch alles recht unsichere Dinge, und von einem auf diesem Wege ermittelten Durchschnittsgewichte des Goldtarì Friedrichs II. lässt sich gar nicht reden. Daraus lässt sich also sicherlich eine Stütze dafür, dass die sizilische libra noch das altrömische Pfund gewesen, nicht zurechtzimmern.

8. Endlich bin ich aber auch mit der Ermittelung des Durchschnittsgewichtes des Augustalis durch Winkelmann (auf 5,30, genauer 5,297 Gramm) nicht ganz einverstanden. Von den 37 Gewichtsangaben, über die Winkelmann verfügte, bewegt sich die grosse Masse zwischen 5,25 und 5,30 Gramm ${ }^{3}$ ); gänzlich fallen aus diesen Rahmen heraus ein Exemplar mit nur 4,93 Gewicht, 2 Exemplare, deren Gewicht auf 5,504 und endlich eins, dessen Gewicht sogar auf 5,796 angegeben wird. Während Winkelmann nun das stark mindergewichtige Exemplar wegen seiner offenbar erheblichen Abnützung bei der Durchschnittsberechnung bei Seite gelassen hat ${ }^{4}$ ), was ich an sich durchaus billige,

1) S. 426 .

2) S. 422 und 426 .

3) Ich setze neben das Gewicht die Zahl der Exemplare in Klammern: $5,30(6), 5,29(2), 5,28(6), 5,27(1), 5,26(6), 5,25(7)$. Dazu je ein Exemplar mit 5,$32 ; 5,24 ; 5,22 ; 5,18$ Gr. Vgl. Winkelmann S. $411 \mathrm{f}$.

4) S, 414 A. 4 . 
hat er jene drei über das Mass hinansgehenden Angaben in die Berechnung einbezogen, obwohl er sich wenigstens bei dem gewichtigsten Exemplar nicht enthalten konnte, der Gewichtsangabe ein Fragezeichen hinzuzufügen ${ }^{1}$ ). Sehr merkwürdig ist es nun, dass diese drei Exemplare sämtlich aus London stammen; gerade von den 4 Exemplaren, die das Britische Museum überhaupt nur besitzt, sollen drei ein $\mathrm{Ge}-$ wicht haben, das alle anderen sonst bekannten Exemplare weit hinter sich lässt. Der Zufall, der hier seine Hand im Spiele haben müsste, erscheint so sonderbar, dass man es gewiss zunächst vorziehen wird, einen Irrtum in der Gewichtsangabe, einen Fehler der Wage oder des Wägenden, anzunehmen. So wird es sich empfehlen, die 4 Londoner Exemplare von der Durchschnittsberechnung wegen des Zweifels, den die in drei Fällen ron dem Normalen stark abweichende Gewichtsangabe hervorrufen muss, ganz auszuschliessen. Wir nehmen an, wir kännten diese Angaben gar nicht; es bleibt dann immerhin noch die stattliche Anzahl von 32 Wägungen übrig. Die auf dieser Grundlage vorgenommene Berechnung ergibt für den Augustalis ein thatsächliches Durchschnittsgewicht von 5,27 Gramm; mit Rücksicht auf die eingetretene Abnutzung muss man natürlich einen Zuschlag machen, um zu dem ursprünglichen, gesetzlichen Durchschnittsgewicht des Augustalis zu gelangen.

9. Unter der eben angegebenen Modifikation des Durchschnittsgewichts des Angustalis würde ich prinzipiell Winkelmann auf der ersten Strecke des Weges, den er zur Bestimmung des sizilischen Gewichtssystems eingeschlagen hat, zu folgen bereit sein; wenn man den Anschluss an das römische Pfund verwirft (womit ich einen Zusammenhang dem Ursprunge nach noch nicht geleugnet haben will), so muss dieser Weg in der That zu einem, wenn auch nicht unbedingt, so doch im wesentlichen sicheren Ergebnis führen. Man würde sich bei einem solchen Ergebnis bescheiden müssen und auch kö»nen, wenn es nicht einen noch zuverlässigeren Weg gäbe, der uns zwar nicht durch die Münzen selbst, aber durch bestimmte und durchaus zuverlässige zeitgenössische Angaben gewiesen wird.

Ich mache zunächst auf folgende Stelle der Münzerordnung aufmerksam: Marcum argenti, secundum quod in regno utimur, est pondus unciarum 8 ad unciam argenti, que uncia ponderat plus quam uncia auri in decima parte, et sic quodlibet marcum argenti, quod ponderat uncias 8 ad uncias argenti, ponderat uncias 8 et tarenos 24 ad racionem uncie auri ${ }^{2}$ ). Die Mark Silber, in 8 Silberunzen zerfallend,

1) S. 412 oben.

2) Acta Imperii ed. Winkelmann I. p. 766 . 
wog also $\left.8^{4}\right|_{5}$ Goldunzen; die Unze Silber wog um $\left.1\right|_{10}$ mehr als die Goldunze; giengen auf die Goldunze 30 Tarì, so giengen 33 Tarì auf die Unze Silber.

Es handelt sich also nur noch darum, ob wir ermitteln können, welcher Mark man sich damals im .Königreich Sizilien bediente. Und über diesen wichtigsten Punkt erhalten wir nun eine durchaus bündige und zuverlässige Auskunft durch den Florentiner Balducei Pegolotti, dessen Pratica di Mercatura im Jahre 1339 abgeschlossen worden ist ${ }^{1}$ ). Sein Buch ist für die Münz-, Mass- und Gewichtsverhältnisse des Mittelalters, Italiens natürlich im besondern, eine überaus wichtige Quelle und ich fürchte nicht, dass man meinen wird, seine Angaben über die in Sizilien geltenden Gewichte könnten nicht als massgebend angesehen werden, da sie nicht der Zeit Friedrichs II. angehören. Gewichte sind Aenderungen nicht so leicht unterworfen wie Münzen; Karl von Anjou hat, wie wir bestimmt wissen, an dem überkommenen Gewichtssystem nichts geändert, obwohl für ihn eine Veranlassung zu solcher Aenderung am ehesten noch vorhanden gewesen wäre, und ebensowenig spricht auch nur das geringste Anzeichen dafür, dass seine Nachfolger im Königreich Sicilien bis zur Zeit Pegolotti's eine solche Aenderung vorgenommen haben.

In ausführlicher Weise vergleicht Pegolotti das in Sizilien geltende Gewichtssystem mit den Systemen der wichtigsten Handelsplätze, die für den Verkehr der Italiener in Betracht kamen. Er gibt zunächst die Grundzüge des Systems selbst: Li 20 grani sono in Cicilia 1 tarì, e gli 30 tarì sono in Cicilia once 1 a peso d'oro, e tarì 33 sono once 1 in Cicilia a peso d'argento; in genauer Uebereinstimmung also mit den erwähnten Angaben der Münzerordnung. Unmittelbar darauf fährt er fort: E rispondono in altre Terre come dirà qui appresso; bei der nun folgenden Vergleichung mit den an anderen Handelsplätzen geltenden Gewichtssystemen aber stellt er an die Spitze: 33 tarì =once 1 in Cologna 2 ).

Damit haben wir, was wir brauchen. Das Gewicht der Kölnischen Mark steht fest; es betrug 233,8 Gramm, das Gewicht der Kölnischen

1) Die Begründung dieses Zeitansatzes habe ich in den Jahrbüchern für Nationalökonomie und Statistik, Band LX (1893) S. 56, Anm. 3 gegeben.

2) Bei Pagnini, Della Decima e di varie altre gravezze. Lisboa e Lucca 1766, tom. III p. 103. Ein Einwand, der möglicherweise erhoben werden könnte, sei hier gleich abgewehrt. Man könnte vielleicht meinen, dass die obige Angabe Pegolotti's nur eine ungefähre sei. Dem widersprechen aber die genauen Angaben in der Tabelle Pegolotti's selbst; z. B.: 'Tarì 34 e grani $13=$ once 1 in Parigi d'argento; tarì 31 e grani $13 \frac{1}{3}=$ once 1 in Siena. Auch ist es sicher nicht zufällig, dass gerade der Vergleich mit dem Kölner Gewicht an der Spitze steht. 
Unze also 29,225 Gr. Diese Unze ist auch die sizilische Silberunze, die 33 Tarì wog; a uf einen Tarì entfallen also 0,8856 Gramm.

Da die sizilische Goldunze 30 Tarì hatte, so ergiebt sich für sie ein Gewicht von 26,568 $\mathrm{Gr}$. und für das aus 12 Unzen bestehende sizilische Goldpfund ein Gewicht von 318,81 Gr. Obwohl dies Ergebnis einer weiteren Bestätigung nicht bedarf, so ist es doch von Interesse, zu vernehmen, dass die noch heute in Sizilien bei Goldschmieden üblichen Gewichte, das Pfund von 317,37, die Unze von 26,45, der Tarì von $0,88 \mathrm{Gr}$. nur sehr geringfügige $\mathrm{Abweichungen} \mathrm{von} \mathrm{diesen}$ Werthen zeigen ${ }^{1}$ ).

Da der Augustalis 6 Tarì wiegen sollte, so entfielen bei genauer Ausprägung auf ihn 5,3136 Gramm. Dies Ergebnis passt vortrefflich zu dem oben aus 32 Exemplaren ermittelten thatsächlichen Durchschnittsgewicht von 5,27 Gr.; die Differenz stellt den Betrag dar, der auf Abnützung zu rechnen ist.

10. Auf Grund der neu gewonnenen Grundlagen lässt sich nunmehr durch einfache Ausführurg der Rechnung der Metallwerth der beiden Unzen, sowie der des Augustalis und des Goldtarì feststellen.

Die uncia tarenorum enthielt unter 30 Gewichtstarì $\left.20^{5}\right|_{12}$ Tarì Feingold (68 Prozent), also, da ein Tarı 0,8856 Gr. wog, 18,081 Gr. Feingold. Rechnet man 1 Gr. Feingold $=2,78$ M., so ergiebt sich danach für die uncia tarenorum ein Metallwerth in Gold von 50,265 Mark.

Sie enthielt ferner $\left.7^{3}\right|_{16}$ Tarì oder 24 Prozent, das sind 6,38 Gramm Feinsilber. Nehmen wir zwischen Gold und Silber die Relation 10:1 an, rechnen also auf $1 \mathrm{Gr}$. Feinsilber $0,278 \mathrm{M}$, so ergiebt sich für die uncia tarenorum ein Metallwerth in Silber von 1,77 M.

$\mathrm{Da}$ der Rest von $\left.2^{19}\right|_{48}$ oder 8 Prozent Kupfer, das sind $2,12 \mathrm{Gr}$. kaum den Zuschlag von 1 Pfennig rechtfertigen würde, so erhalten wir damit den Metallwerth der uncia tarenorum mit $52 \mathrm{M}$. 3 Pfennigen. Auf den normal ausgeprägten einzelnen Goldtarì würde danach ein Metallwerth von 1,73 Mark entfallen.

Die uncia augustalium enthielt unter 24 Gewichtstarì $=21,254 \mathrm{Gr}$. $20 y_{2}$ Tarì, das sind 85,4 Prozent Feingold $=18,15$ Gramm; dieses in ihr enthaltene Quantum Feingold hat einen Metallwerth von 50,46 M.

Sie enthielt ferner $\left.2^{10}\right|_{16}$ Tarì $=11$ Prozent Feinsilber. Während ihr Kupfergehalt ( $\left.7\right|_{8}$ Tarì $=3,6$ Prozent) so gering war, dass or für die Werthberechuung gar nicht in Betracht kommen kann, hatten die 2,34 Gramm Feinsilber, die den $\left.2^{10}\right|_{16}$ Tarì entsprechen, einen Metallwerth von $0,65 \mathrm{M}$.

i) Winkelmann S. 414 . 
Danach ergiebt sich für die uncia augustalium ein Metallwerth von 51,11 M., während a uf den einzelnen A ugustalis als den vierten Theil derselben ein Metallwerth von 12,78 M. entfällt.

Mithin blieb die uncia augustalium an Metallwerth hinter der uncia turenorum um 92 Pfennige, der einzelne Augustalis hinter den $71_{2}$ Goldtarì, denen er im Werthe gleichgesetzt war, um 23 Pfennige zurück, das sind etwa 1,7 Prozent.

Für die Unze reinen Goldes endlich würde sich ein Werth von 73,86 M., für den einzelnen Tarì ein Werth von 2,46 M. ergeben. Danach sind also $\left.{ }^{3}\right|_{8}$ Tarì an Feingold, die wir oben als Differenz zwischen dem Metallwerthe der uncia augustalium und der uncia tarenorum ermitteit hatten, gleich 92 Pfennigen und die Rechnung stimmt.

11. Es bleiben uns noch die Fragen nach dem Kostenwerthe und dem Verkehrswerthe der sizilischen Goldunze und ihrer ausgeprägten Theile zu beantworten.

Das uns hiefür zur Verfügung stehende Material besteht einmal in der oben angeführten Stelle der Münzerordnung, die die Höhe der von Privaten, die für eigene Rechnung prägen liessen, $\mathrm{zu}$ erhebenden Beträge festsetzt: $\left.15^{1}\right|_{2}$ Gran Abgabe an den Fiskus und $\left.4^{1}\right|_{2}$ Gran als Ersatz für die Prägekosten, falls nicht etwa Bestreitung der erforderlichen Ausgaben, Lieferung der Materialien u. dgl. durch den Auftraggeber selbst erfolgte; zweitens in folgenden Bemerkungen, die an Schluss der Münzerordnung im allgemeinen und des die Aufschrift: ,De sicla Messane tragenden Passus im besonderen stehen ${ }^{1}$ ):

Dncia tarenorum auri sicle valet ad sumptum tarenos 28 et duas partes unius grani.

Uncia augustalium valet ad sumptum tarenos 27 et grana 18.

Was bedeutet zunächst, valet ad sumptum'? Winkelmann bezieht es auf den Metallwerth der Unze ${ }^{2}$ ), doch scheint es, dass er die Beifügung ,ad sumptum' nicht beachtet hat. ,Die Unze kommt der Münze in Bezug auf den Aufwand zu stehen', so hätte man sich schwerlich ausgedrückt, wenn es sich nur um den Einkaufspreis des Metalls gehandelt hätte. Es handelte sich für die Münze um Feststellung des Gewiunes, der an die Regierung abzuführen war; wesentlicher als die Differenz zwischen Einkaufspreis und Nominalwerth und von praktischer Bedeutung für sie musste die Differenz zwischen dem Nominalwerthe und dem Selbstkostenwerthe der Unze sein. Auf diesen Kostenwerth also meine ich den Ausdruck, valet ad sumptum' beziehen zu

1) Acta Imperii I, 767.

2) S. 434 . 
müssen, den Begriff ,Kostenwerth' nicht in moderner Feinheit, sondern nur als die aus dem Einkaufspreise und der Gesammtheit der unmittelbaren Herstellungskosten gewonnene Summe genommen. Auch würde ich diesen am Schluss der Münzerordnung angegebenen Kostenwerth nicht gerade als eine völlig unveränderliche Grösse angesehen, eher als einen Durchschnittswerth, den die Beamten der Münze von Messina aufgestellt haben, um ihrerseits einen festen Anhalt zu haben.

In unserer Auffassung des ,valet ad sumptum' werden wir auch dadurch bestärkt, dass die Differenz in den Metallwerthen der uncia tarenorum und uncia augustalium nicht übereinstimmt mit der Differenz, die die Werthe der beiden Unzen an dieser Stelle der Münzerordnung aufweisen. Die Differenz in dem Kostenwerth der beiden Unzen, $\left.2^{2}\right|_{3}$ Gran, erweist sich als wesentlich niedriger als die Differenz ihrer Metallwerthe. Das würde sich daraus erklären lassen, dass die Kosten der Prägung der Augustales wesentlich höher waren als für die Prägung der Goldtarì. Bei den Augustales, die nicht zugewogen wurden, die mit ihrem feststehenden, zuverlässigen Werthe von Hand zu Hand gehen sollten, war ein ganz anderer Grad von technischer Sorgfalt, Kunst und Exaktheit und damit auch ein grösserer materieller Aufwand erforderlich als bei der Prägung von Goldtarì, bei denen es wesentlich nur auf die Richtigkeit der Legierung ankam; mit Recht hebt Winkelmann bei diesen die Rohheit der Arbeit, die sehr von der der Augustalen absticht, besonders hervor ${ }^{1}$ ). Nun könnte man meinen, dass die Regierung danı für die Ausprägung von Augustalen von Privaten eine höhere Prägegebühr erhoben haben müsste. Das geschah indessen nicht, vermutlich weil man an dem seit Alters feststehenden Satze (consuevit curia recipere) nicht gern ändern wollte, dann aber auch, weil der Gewinn der Regierung auch so noch hoch genug blieb und weil man wohl durch Gewährung eines etwas höheren Gewinnantheils Private veranlassen wollte, ihr Gold lieber in der neu geschaffenen Goldmünze ausprägen zu lassen als in den altherkömmlichen und deshalb im Verkehr zunächst noch beliebteren Goldtarì. Liess die Regierung auf eigene Rechnung prägen, so hatte sie bei der Prägung von Augustalen immer noch einen etwas höheren Gewinn als bei der Prägung von Goldtarì. Dass man den Metallwerth der uncia augustalium nicht ganz unerheblich niedriger ansetzte als den der uncia tarenorum, würde sich also daraus erklären, dass man von vorneherein auf die höheren Prägekosten Rücksicht nahm und bei eigener Prägung auch noch einen um ein Geringes höheren Gewinn machen wollte als

1) S. 425 , vgl. auch 424 . 
bei der Prägung der Goldtarì. Treffen diese Ausführungen das Richtige, so wird man nur für die uncia tarenornm den Kostenwerth dadurch ermitteln dürfen, dass man ihrem Metallwerthe die von Privaten erhobene Prägegebühr von $\left.4^{1}\right|_{2}$ Gran zuschlägt. Ist die Gebühr, was an sich nicht unwahrscheinlich, zu hoch gegriffen, so kann das doch wohl nur eine Kleinigkeit ausmachen, da den Kaufleuten das Recht der unmittelbaren Bestreitung der erforderlichen Ausgaben eingeräumt war. Für die uncia angustalium dürfen wir nicht so verfahren, weil bei dieser die Prägekosten höher gewesen sein müssen; ihren Kostenwerth können wir nur durch Abzug der aus der Münzerordnung sich ergebenden Differenz im Betrage von $\left.2^{2}\right|_{3}$ Gran von dem Kostenwerthe der uncia tarenorum ermitteln.

Der Nennwerth oder Verkehrswerth, der ja für beide Unzen gleich hoch normiert war, ergiebt sich dann in einfacher Weise daraus, dass wir dem Kostenwerthe (Metallwerth und Prägekosten) der uncia tarenorum den Schlagschatz, d. h. die Differenz zwischen dem Nennwerthe von 30 Tarì und dem von der Münzerordnung angegebenon Kostenwerthe von 28 Tarì ${ }_{\mid 3}$ Gran zuschlagen.

Danach ist also die uncia tarenorum oder 600 Gran Nennwerth $=52,03$ Mark (Metallwerth) $+\left.4\right|_{2}$ Gran Nennwerth (Prägegebühr) $+\left.39^{1}\right|_{3}$ Gran Nennwerth (Schlagschatz). Es sind also $\left.556^{1}\right|_{6}$ Gran Nennwerth $=52,03 \mathrm{M}$., so dass auf einen Gran Nennwerth 0,0935 M. entfallen.

Nun ergiebt sich alles andere leicht. Die Prägegebühr mit $\left.{ }^{4}\right|_{2}$ Gran Nennwerth betrug 42 Pfennige, der Schlagschatz. mit $\left.{ }^{39^{1}}\right|_{3}$ Gran Nennwerth 3,68 M. auf die uncia tarenorum. Der einzelne Goldtarì hatte einen Nennwerth von 1,87 , die uncia tarenorum einen solchen von $56,13 \mathrm{M}$.

Dieser Werth war auch der Nennwerth der uncia augustalium. Ihr Kostenwerth aber war um $\left.2^{2}\right|_{3}$ Gran, das sind 25 Pfennige, geringer als der der uncia tarenorum; er betrug bei dieser 52,45 Mark, bei der uncia augustalium also 52,20 M. Der Fiskus machte bei der Prägung von Augustalen einen Gewinn von 3,93 M. auf die Unze.

Der einzelne Augustalis endlich hatte demnach bei einem Metallwerthe von 12,78 M. einen Kostenwerth von 13,05 M. und einen Nennwerth von 14,03 Mark, während sein Aequivalent in Goldtarì $\left(=\left.71\right|_{2}\right.$ Tari $)$ bei einem Kostenwerthe von 13,11 einen Metallwerth von $13,01 \mathrm{M}$. besass.

12. Der bequemeren Uebersicht wegen stelle ich nunmehr noch die Ergebnisse für den Goldtarì, den Augustalis, das Aequivalent des- 
selben gleich $\left.7^{11}\right|_{2}$ Goldtarì, die uncia tarenorum und die uncia augustalium tabellarisch zusammen.

\begin{tabular}{|c|c|c|c|c|c|c|}
\hline & $\begin{array}{c}\text { Gewicht } \\
\text { in Gr. }\end{array}$ & Goldgehalt & Silbergehalt & $\begin{array}{l}\text { Metall- } \\
\text { werth } \\
\text { in } \mathrm{M} \text {. }\end{array}$ & $\begin{array}{c}\text { Kosten. } \\
\text { werth } \\
\text { in } \mathbf{M} \text {. }\end{array}$ & $\begin{array}{l}\text { Nenn- } \\
\text { werth } \\
\text { in } M .\end{array}$ \\
\hline Tarì : & 0,8856 & $\begin{array}{l}0,603 \mathrm{Gr} .= \\
1,675 \mathrm{M} .\end{array}$ & $\begin{array}{l}0,213 \mathrm{Gr} .= \\
0,059 \mathrm{M} .\end{array}$ & 1,73 & 1,748 & 1,87 \\
\hline $71 / 2$ Tarì: & 6,642 & $\begin{array}{c}4,52 \mathrm{Gr} .= \\
12,565 \mathrm{M} .\end{array}$ & $\begin{array}{l}1,595 \mathrm{Gr} .= \\
0,44 \mathrm{M} .\end{array}$ & 13,01 & 13,11 & \multirow[b]{2}{*}{14,03} \\
\hline Augustalis : & 5,314 & $\begin{array}{l}4,54 \text { Gr. }= \\
12,615 \mathrm{M} .\end{array}$ & $\begin{array}{l}0,585 \mathrm{Gr} .= \\
0,16 \mathrm{M} .\end{array}$ & 12,78 & 13,05 & \\
\hline uncia taren.: & 26,568 & $\begin{array}{l}\overline{18,08 \mathrm{Gr} .}= \\
50,26 \mathrm{M} .\end{array}$ & $\begin{array}{l}6,38 \mathrm{Gr} .= \\
1,77 \mathrm{M} .\end{array}$ & 52,03 & 52,45 & \multirow{2}{*}{56,13} \\
\hline uncia august. & 21,254 & $\begin{array}{l}18,15 \text { Gr. }= \\
50,46 \text { M. }\end{array}$ & $\begin{array}{l}2,34 \text { Gr. }= \\
0,65 \mathrm{M} .\end{array}$ & 51,11 & 52,20 & \\
\hline
\end{tabular}

Am bedeutendsten ist, äusserlich genommen, die Abweichung meiner Ergebnisse von denen Winkelmanns bezüglich des Metallwerthes der beiden Unzen, also gerade bezüglich des wichtigsten Punktes; er stellt sich bei der uncia augustalium um 1,77 M. niedriger, bei der uncia tarenorum dagegen um $0,43 \mathrm{M}$. höher, als Winkelmann berechnet hatte. Damit ist zugleich das gegenseitige Werthverhältnis der beiden Unzen das umgekehrte geworden, als Winkelmann angenommen hatte; nicht die uncia augustalium war 1,22 M. mehr werth, wie die uncia tarenorum, sondern umgekehrt, die uncia tarenorum hatte einen um 92 Pfennige höheren Metallwerth als die uncia augustalium. Damit stimmen die Angaben der Münzerordnung überein; die Aenderungen Winkelmanns an derselben haben sich als nicht stichhaltig erwiesen. Als nicht begründet haben wir auch das Zurückgehen Winkelmanns auf das altrömische Pfund erkannt; durch den Nachweis, dass die sizilische Silberunze, die die Goldunze um $\left.1\right|_{10}$ an Gewicht übertraf, mit der Kölnischen Unze identisch gewesen, glauben wir die Berechnung der Gewichte des sizilischen Königreiches auf eine völlig sichere Basis gestellt zu haben; das auf Grund der zahlreichen, durch das Verdienst Winkelmanns für den Augustalis beigebrachten Gewichtsangaben ermittelte Durchschnittsgewicht dieser Goldmünze haben wir in bester Uebereinstimmung mit dieser Basis gefunden. Dagegen müssen wir zugestehen, dass auch unserem Resultat eine gewisse Unsicherheit anhaftet wegen der Berechnung des Silberwerthes: es ist zuzugeben, dass die von uns angenommene Werthrelation zwischen Gold und Silber von 10:1 von der bei den Edelmetallkäufen der Münze wirklich in Geltung gewesenen, sei es nach oben oder nach unten hin, abweichen kann. Iudessen ist diese Fehlergrenze nicht zu 
weit zu ziehen; selbst bei der uncia tarenorum mit ihrem bedeutenden Silbergehalt würde der Fehler nur 0,16 M. betragen, wenn wir die Werthrelation bis auf 11:1 hinaufrücken wollten.

Bezüglich des Nennwerthes der beiden Unzen oder ihres Verkehrswerthes, wie Winkelmann ihn bezeichnet, weicht mein Ergebnis in der Weise ab, dass es das Winkelmann'sche um 77 (76) 1) Pfennige übertrifft. Man sollte eigentlich eine stärkere Abweichung erwarten; dass sie nicht eingetreten ist, liegt daran, dass die mancherlei Irrthümer der Winkelmann'schen Berechnung sich zum Theil in entgegengesetzter Richtung bewegen und so ausgleichend gewirkt haben.

Die Fehlerquelle, deren Vorhandensein wir bei Berechnung des Metallwerthes festgestellt haben, muss natürlich die Berechnung des Nennwerthes in gleicher Weise beeinflussen. Eine zweite Unsicherheit liegt aber hier noch insofern vor, als es zweifelhaft ist, ob sich die Prägegebühr, die bei privater Prägung erhoben wurde, mit den wirklichen Prägekosten deckte. Dass es nicht der Fall war bei der Ausmünzung von Augustalen, dass hier vielmehr die Kosten höher waren als die Gebühr, glauben wir nachgewiesen zu haben; deshalb haben wir auch zur Berechnung des Nennwerthes der uncia augustalium diese Gebühr nicht herangezogen. Die altherkömmliche Prägegebühr war ursprünglich nur für die uncia tarenorum angesetzt, und zwar wahrscheinlich ein wenig ${ }^{2}$ ) zu hoch. Um diesen höheren Betrag würde also der von uns berechnete Nennwerth zu verringern sein; wenn es sich hier, bei einer Prägegebühr von 42 Pfennigen, um 10 Pfennige handeln sollte, so ist das jedenfalls schon sehr hoch gegriffen.

Grösser würde der Fehler sein, wenn unsere Auffassung des valet ad sumptum der Münzerordnung sich als irrig erweisen sollte; dann würden etwa 40 Pfennige von unserem Nennwerthe in Abzug zu bringen sein; indessen halte ich diese Befürchtung für völlig ausgeschlossen, schon weil dann die Verschiedenheit der Differenz zwischen dem wirklichen Metallwerthe der beiden Unzen von der Differenz zwischen den beiden Angaben der Münzerordnung für das, valet ad sumptum' ganz unerklärbar sein würde.

So glaube ich annehmen zu können, dass ich, selbst den ungünstigsten Fall gesetzt, bei der Angabe des Nennwerthes möglicherweise bis zur Grenze von etwa 26 Pf. bei der uncia tarenorum, von etwa 16 Pf. bei der uncia augustalium zu hoch, oder nach der anderen

1) Dies Schwanken hat darin seinen Grund, dass W. den Verkehrswerth der unc. tar. mit 55,37 , den der unc. aug. mit 55,36 angiebt. $S$. 440 .

2) Vgl. ob. S. 560 . 
Seite bis zur Grenze von etwa 10 Pf. zu niedrig abgekommen sein kồnnte. Wenn ich diese möglichen Fehlergrenzen ausdrücklich anerkenne, so scheint mir doch keine ausreichende Veranlassung vorzuliegen, an meinem Ergebnis selbst eine Aenderung vorzunehmen. Bezüglich des einzelnen Augustalis verringert sich diese Fehlergrenze naturgemäss auf den vierten Theil.

Um mit einer allgemeinen Bemerkung zu schliessen, so möchte ich meine Meinung dahin aussprechen, dass Kaiser Friedrich, als er die Prägung der Augustalen anordnete, damit die Einführung eines neuen Münz s y stems nicht beabsichtigt hat; nach wie vor wird genau in derselben Weise, nicht bloss von Privaten, sondern von der Regierung selbst nach Unzen, Tarì und Gran gerechnet. Danach theile ich auch die Anschauung Winkelmanns nicht, dass Friedrich II. damit die Goldwährung des sizilischen Königreichs in Verwirrung gebracht habe; ich kann auch kein Unding darin sehen, dass nun zwei gesetzliche Zahlungsmittel neben einander vorhanden waren ${ }^{1}$ ), deren Einheiten im Werthrerhältnisse von $1:\left.7^{1}\right|_{2}$ gestanden hätten. Die Neuerung Friedrichs bedeutet für den Verkehr einen Fortschritt zum Besseren, eine Erleichterung; während die Goldmünzen des Königreichs bisher nur nach ihrem Gewicht kursieren konnten, sobald es sich irgend um grössere Beträge handelte, waren nunmehr trefflich ausgeprägte Goldmünzen vorhanden, deren Werth ein genau bestimmter und vollkommen zuverlässiger war, so dass die einfache Zuzählung durchaus genügte. In dieser blossen Thatsache lag ein Vortheil; der dadurch nicht aufgehoben wurde, dass die alten Münzen, die man zuwägen musste, im Gebrauch blieben. Und was das Werthverhältnis der beiden Münzen anbetrifft, so muss man seine Aufmerksamkeit eben in erster Linie auf die Rechnungs einheit richten. Diese war die Goldunze und nicht der Tarì; durch die Ausmünzung von Viertelunzen und Achtelunzen konnte die Rechnung nach Unzen nicht in Verwirrung gebracht werden. Auch die Meinung scheint mir nicht ausreichend begründet, dass die neue Goldmünze sich niemals recht eingebürgert habe; besitzen wir doch Beweise, dass der Augustalis in den sechziger Jahren des 13. Jahrhunderts selbst in Frankreich stark im Umlauf war, und als eine Erinnerung an die Goldmünzen seines Vorgängers ist es zu betrachten, wenn Heinrich VII. im Jahre 1311 für Mailand die Ausprägung von Augustarii anordnete ${ }^{2}$ ).

1) S. 439 ; vgl. $404,417,429$.

2) v. Inama-Sternegg a. a. 0. 24. Im übrigen vgl. die eigenen Angaben Winkelmanns S. $430 \mathrm{f}$. 
Im übrigen bin auch ich der Meinung Winkelmanns, dass für die Art der Gestaltung der neuen Goldmünzen die imperialistischen Tendenzen des Herrschers massgebend gewesen sind; nur hat sich die Regierung um dieser Tendenzen willen bei der Ausprägung von Augustalen kein finanzielles Opfer auferlegt, vielmehr hat sie die Gelegenheit benützt, um ihren Gewinn bei der Goldausmünzung, wenn auch nicht gerade in erheblicher Weise, noch weiter zu steigern. 